\title{
MEMBERSHIP OF COMMISSIONS AND ALPHABETICAL LIST OF MEMBERS
}

Reference is made to Appendix II.

\section{LIST OF COUNTRIES ADHERING TO THE UNION 45}

The following is a list of the 47 countries that adhered to the Union in September 1973, giving also the year of admission, the approximate number of Members, and the Adhering Organizations.

\section{Country}

Arab Republic

of Egypt

Argentina

Australia

Austria

Belgium

Brazil

Bulgaria

Canada

Chile

Colombia

Cuba

Czechoslovakia

Denmark

Finland

France

rifeimivis, F.R.

G.D.R.

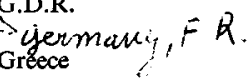

Hungary

India

$\because \quad$ Iran

Yrełand

1476

Israel

Italy

Japan

Korea (DPR)

Korea (Republic)

Mexico
Year

Members

1925

1927

1939

1955

1920

1961

1957

1920

1947

1967

1970

1922

1922

1948

1920

1951

1951

1920

1947

1964

1969

1947

1954

1921

1920

1961

1973

1921

$0 \quad 0$

4040

$20 \quad 20$

IY1 141

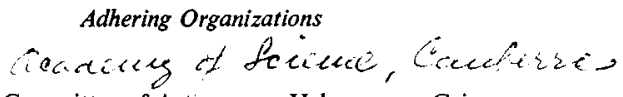

1110 National Committee of Astronomy, Helwan near Cairo

2627 ^ 6 Comité Nacional de Astronomía, ba Plata : Late

6465, Australian National Committee for Astronomy, Sydney.

69 1918 Österreichische Akademie der Wissenschaften, Wien

$\$ 86158$ Académie Royale de Belgique, Bruxelles,

Koninklijke Academie van België, Brussel

1414 Comissão Brasileira de Astronomia (CBA), Conselho

Nacional de Pesquisas, Rio de Janeiro

1010 Bulgarian Academy of Sciences, Sofia

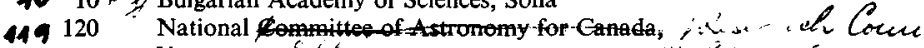

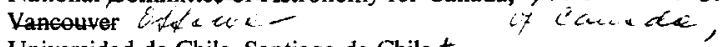

77 Universidad de Chile, Santiago de Chile $f$

11 Academia Colombiana de Ciencias Exactas, Físicas y Naturales, Bogotá

Instituto de Astronomía de la Academia de Ciencias de Cuba, La Habana

National Committee of Astronomy of Czechoslovakia, Czechoslovak Academy of Sciences, Praha

Danish National Committe for Astronomy, det Konge-

like Danske Videskabernes Selskab, København

1313 Fcademy of Sciences and Letters, Helsinki fu «ce

225226225 Comité National Français d'Astronomie, Académie des Sciences, Paris

15 154151 Rat Westdeutscher Sternwarten 2w-Aünetren 15 zew

34 35 Nationalkomitee für Astronomie der DDR, Akademie der Wissenschaften der DDR, Berlin

2525 Greek National Committee for Astronomy, Academy of Athens, Athens

1314 - Hungarian Academy of Sciences, Budapest

5757 Indian National Gommttee of Astronomy, Indian fis equa $Q_{i}$ National Science Academy, New Delhi ve. \&

1 Tehran University, Tehran teveice.

- 9 National Committee of Astronomy, Royal Irish Academy, . Dublin

2121 The Israel Academy of Sciences and Humanities, Jerusalem

10111 Consiglio Nazionale delle Ricerche, Roma

National Committee of Astronomy of Japan, Science

Council of Japan, Tokyo

33.4 Academy of Sciences, Phyongyang

44 Korean Astronomical Society, Seoul - Luce ve

2121 Universidad Nacional de Mexico, Mexico D.F

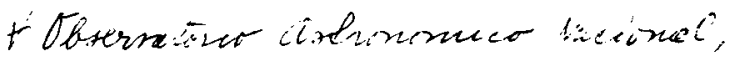


Country

Netherlands

New Zealand

Norway

Poland

Portugal

Roumania

South Africa

Spain

Sweden

Switzerland

Taiwan

Turkey

United Kingdom

Uruguay

U.S.A.

U.S.S.R.

Vatican City State

Venezuela

Yugoslavia

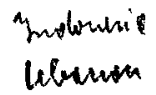

Year

1922

1964

1922

1922

1924

1928

1938

1922

1925

1923

1959

1961

1920

1970

1920

1935

1932

1953

1935

\section{Members}

Adhering Organizations

86 8786 Koninklijke Nederlandse Academie van Wetenschappen, Amsterdam $\begin{array}{rr}9 & 9 \\ 14 & 15 \\ 49 & 50 \\ 14 & 15\end{array}$ $\boldsymbol{l}_{\text {Royal Society of New Zealand, Wellington }}$ 14 Det Norske Videnskaps-Akademii, Oslo 44 Polska Akademia Nauk, Warszawa

1415 Secçāo Portuguesa das Uniōes Internacionais Astronó18 mica, Geodésica e Geofísica, Lisboa 11 13 19 Council for Scientific and Industrial Research, Pretoria 1218 Comisión Nacional de Astronomía, Madrid 5051 Kungl. Vetenskapsakademien, Stockholm 26 26 Schweizerische Naturforschende Gesellschaft, Genf 1010 Academia Sinica, Taipei

2222 Türk Astronomi Dernegi, Istanbul $26 \mathbf{2 7 7} 27$ iThe Royal Society, London

- 0 Comité Nacional de Astronomía, Montevideo 404920 907 The National Academy of Sciences, Washington $383394 ; 93$ Academy of Sciences of the U.S.S.R., Moscow 67 Pontificia Accademia delle Scienze, Città del Vaticano 33 Academia de Ciencias Física, Matemáticas y Naturales, Caracas

2020 Savez društ va matematičara, fizičara i astronoma Jugoslavije, Z Nin fed

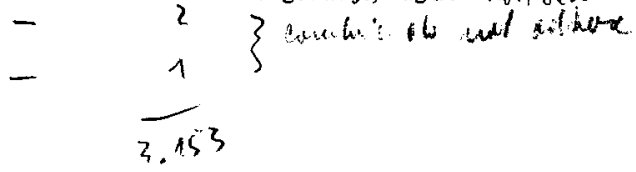

